\section{A "TRICK" for the Design of FIR Half-Band Filters}

\author{
P. P. VAIDYANATHAN AND TRUONG Q. NGUYEN
}

\begin{abstract}
Based on a well-known property of FIR half-band filters, this correspondence shows how the design time for equiripple half-band filters can be reduced by a considerable amount. The observation which leads up to this improved procedure also places in evidence new implementation schemes, which simultaneously ensure low passband and stopband sensilivities. Extension of the method to $M$ th-band filter design is also outlined.
\end{abstract}

\section{INTRODUCTION}

Linear-phase FIR half-band filters have found several applications in the past [1], [4]. For instance, in the design of sharp cutoff FIR filters, a multistage design based on half-band filters is very efficient [2]. The efficiency of half-band filters derives from the fact that about 50 percent of the filter coefficients are zero, thus, cutting down the implementation cost. Half-cost filters have also been used in multirate filter bank applications, either directly or indirectly [3], [4].

Let $H(z)$ denote the transfer function of a (linear-phase, FIR) half-band filter of order $N-1$

$$
H(z)=\sum_{n=0}^{N-1} h(n) z^{-n}, \quad h(n) \text { real. }
$$

These filters are restricted to be of Type 1 (i.e., $N-1$ is even and $h(n)=h(N-1-n)[12])$. The frequency response is thus of the form $H\left(e^{j \omega}\right)=e^{-j \omega N-1 / 2} H_{0}\left(e^{j \omega}\right)$, where $H_{0}\left(e^{j \omega}\right)$ represents the real-valued amplitude response. A typical plot of $H_{0}\left(e^{j \omega}\right)$ is shown in Fig. 1, assuming an equiripple type of design. There is a symmetry with respect to the half-band frequency $\pi / 2$, i.e., the band edges are related as

$$
\omega_{p}+\omega_{S}=\pi
$$

and the ripples are related as

$$
\delta_{1}=\delta_{2}=\delta
$$

In view of this symmetry, the impulse response $h(n)$ satisfies

$$
h(n)=\left\{\begin{array}{ll}
0, & n-\frac{N-1}{2}=\text { even and nonzero } \\
\frac{1}{2}, & n=\frac{N-1}{2}
\end{array} .\right.
$$

The simplest way to design equiripple half-band filters is to invoke the widely used McClellan-Parks algorithm [5] with the specifications satisfying (2a) and (2b). (If equiripple nature is not a requirement, then window designs are the fastest [2].) The resulting filter satisfies (3) with reasonable accuracy. The only

Manuscript received July 18, 1986; revised September 29, 1986

The authors are with the Department of Electrical Engineering, California Institute of Technology, Pasadena, CA 91125. This work was supported in part by the National Science Foundation under Grant ECS 84-04245, in part by Caltech's programs in Advanced Technology grant, sponsored by Aerojet General, General Motors, GTE and TRW, and in part by the Naval Oceans Systems Center, through a subcontract by the San Diego State University Foundation.

IEEE Log Number 8611707.

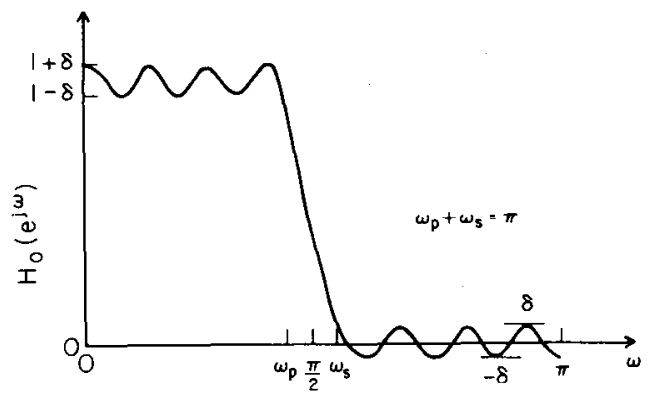

Fig. 1. Typical amplitude response of a half-band FIR filter.

disadvantage with this procedure is that those coefficients which are supposed to satisfy (3) are treated as unknowns in the optimization, and, accordingly, the design time is longer than necessary.

In this correspondence, we first indicate a method (the "halfband trick") for considerably reducing the design time by exploiting the partial knowledge (3) about the impulse response coefficients. The technique also leads to a structural interpretation of half-band filters, which enables us to implement these filters in such a way that, if the structure has low passband sensitivity, then it automatically has low stopband sensitivity as well. (This is significant in view of the fact that low passband and low stopband sensitivities are often conflicting requirements [6].) We conclude by indicating how the ideas can be extended for $M$ thband filters [7] (which find application in decimation and interpolation filters). Design examples are presented demonstrating the significant features of the results.

\section{The HALF-BAND Design Trick}

First notice that, in view of (3), we can always assume $(N-1) / 2$ to be odd. (Indeed, if $(N-1) / 2$ were even, then (3) would imply $h(0)=h(N-1)=0$; by redefining $h(1)$ to be $h(0)$, we can cut down the order to $N-3$.) Given the specifications $\omega_{p}, \omega_{S}$, and $\delta$, let us first design a one-band prototype linear-phase filter $G(z)$ of order $(N-1) / 2$ with specifications as shown in Fig. 2. $G(z)$ has a zero at $\omega=\pi$, since $(N-1) / 2$ is odd [12]. Its passband extends from 0 to $2 \omega_{p}$ and the transition band is from $2 \omega_{p}$ to $\pi$. If we now define

$$
H(z)=\frac{G\left(z^{2}\right)+z^{-N-1 / 2}}{2}
$$

then $H(z)$ is a half-band filter, with specifications as in Fig. 1. The conditions (2a), (2b), and (3) are satisfied exactly. The impulse response of $H(z)$ is evidently related to that of $G(z)$ by

$$
h(n)= \begin{cases}\frac{1}{2} g\left(\frac{n}{2}\right), & n \text { even } \\ 0, & n \text { odd } \neq \frac{N-1}{2} . \\ \frac{1}{2}, & n=\frac{N-1}{2}\end{cases}
$$

$G(z)$ can be designed with the help of the McClellan-Parks program. This design time is considerably lower than the time required to design $H(z)$ directly, since the order of $G(z)$ is only $(N-1) / 2$. Moreover, for large $N-1$, the design-accuracy is better. 


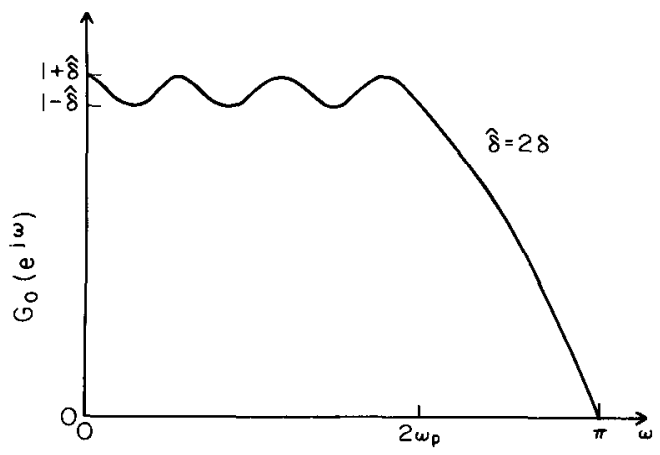

Fig. 2. Amplitude response of $G(z)$.

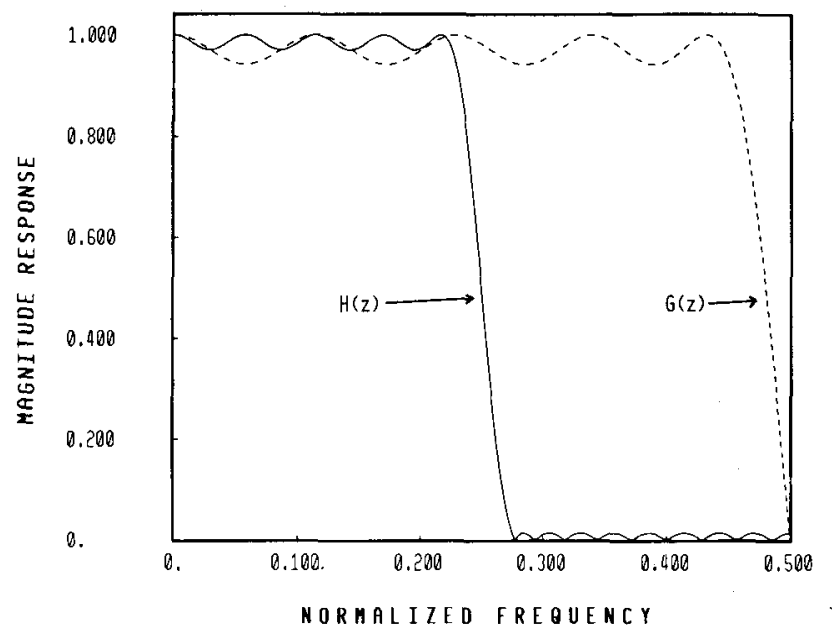

Fig. 3. Ex. 1: Magnitude response plot of $G(z)$ and the half-band filter.

TABLE I

COMPARISON OF THE IMPROVED AND DIRECT METHODS IN Half-Band Filter Designs.

\begin{tabular}{lcccc}
\hline \hline$N-1$ & $\Delta f$ & $\delta_{1}$ & $\begin{array}{c}\text { CPU Time } \\
(\text { Bec.) }\end{array}$ & $\begin{array}{c}\text { CPU Time } \\
\text { (8ec.) }\end{array}$ \\
18 & .1 & $1.135 \times 10^{-3}$ & 0.6 & 1.9 \\
30 & .1 & $1.350 \times 10^{-3}$ & 1.0 & 4.3 \\
42 & .1 & $1.715 \times 10^{-4}$ & 1.4 & 6.4 \\
50 & .05 & $3.550 \times 10^{-3}$ & 1.8 & 9.4 \\
62 & .05 & $1.255 \times 10^{-3}$ & 2.4 & 14.9 \\
82 & .05 & $2.275 \times 10^{-4}$ & 3.8 & 25.2 \\
\hline
\end{tabular}

$N-1$ is the required order for peak passband ripple $\delta_{2}$ and transition width $\Delta f$.

Example 1: A half-band linear phase FIR filter of order $N-1$ $=34$, and $\omega_{p}=0.45 \pi$ is designed using the above method. The magnitude responses of $G\left(e^{j \omega}\right)$ and $H\left(e^{j \omega}\right)$ are shown in Fig. 3. To demonstrate the saving in design time of the above method, we compare the design time of this method with the conventional method for several half-band filters with various specifications. Table I summarizes the results. For higher orders, the savings is quite significant (about a factor of 7 for $N-1=82$ ).

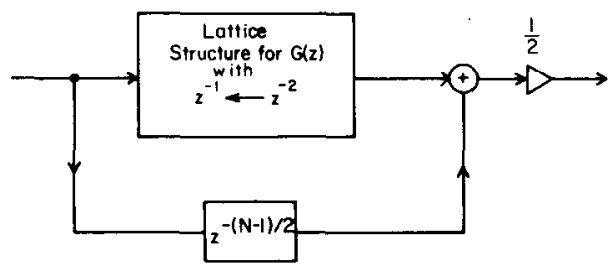

Fig. 4. Structure for half-band filter.

\section{Low Sensitivity Structures for Half-Band FILTERS}

It is well known [6], [8] that a digital filter structure having low passband sensitivity does not necessarily have low stopband sensitivity, and vice versa. The coefficient-sensitivity problem in FIR structures has been analyzed in the past [6], [9], [10]. Based on the notion of structural passivity, certain lattice structures are proposed in [10] which can be used to synthesize low-sensitivity structures for any arbitrary FIT transfer function.

The lattice structures in [10] satisfy two crucial properties: first, they provide very low passband sensitivity; second, if the transfer function has linear phase, this linearity is maintained even when the lattice coefficients are quantized. Now assume that we first implement the one-band filter $G(z)$ using such a structure. Then $G(z)$ has how passband sensitivity. When the lattice coefficients are quantized, the magnitude response of the transfer function $G_{q}(z)$ remains very close to $G(z)$. Since $G_{q}(z)$ retains linear phase and has odd order $(N-1) / 2$, it continues to have the zero at $\omega=\pi$ despite quantization. Suppose we realize $H(z)$ from this structure for $G(z)$, exactly as suggested by (4) (see Fig. 4). Then the stopband response of $H(z)$ is exactly an image of its passband response, even if the coefficients of the lattice are quantized! Thus, $H_{q}(z)$ (the response of the quantized lattice) continues to remain a half-band filter and has low passband as well as stophand sensitivities.

Example 2: A half-band filter $H(z)$ with $\omega_{p}=0.45 \pi, \omega_{S}=$ $0.55 \pi$, and with stopband attenuation of about $86 \mathrm{~dB}$ was implemented as in Fig. 4. The required order was $N-1=102(G(z)$ has order 51). The internal details of the lattice structure used to implement $G(z)$ are not relevant here and can be found in [10]. The lattice coefficients were quantized to 5 bits in canonical sign digit code, and the resulting response is as shown in Fig. 5(a). A direct-form implementation of $H(z)$ with coefficients quantized to 5 bits in canonical sign digit code has response as in Fig. 5(b). It is evident that the lattice structure has good passband and stopband sensitivities.

\section{Extension to THE Design of $M$ th Band Filters}

An $M$ th band linear-phase (low-pass) FIR filter [12] is a Type 1 filter $H(z)$ with amplitude response $H_{0}\left(e^{j \omega}\right)$ as shown in Fig. 6. The impulse response $h(n)$ has one out of $M$ samples equal to zero; the main properties equal to zero; the main properties can be summarized as follows:

$$
\begin{gathered}
\omega_{p}+\omega_{S}=\frac{2 \pi}{M} \\
\delta_{1} \leq(M-1) \delta_{2} \\
h(n)= \begin{cases}0, & n-\frac{N-1}{2}=\text { nonzero multiple of } M \\
\frac{1}{M}, & n=\frac{N-1}{2}\end{cases}
\end{gathered}
$$




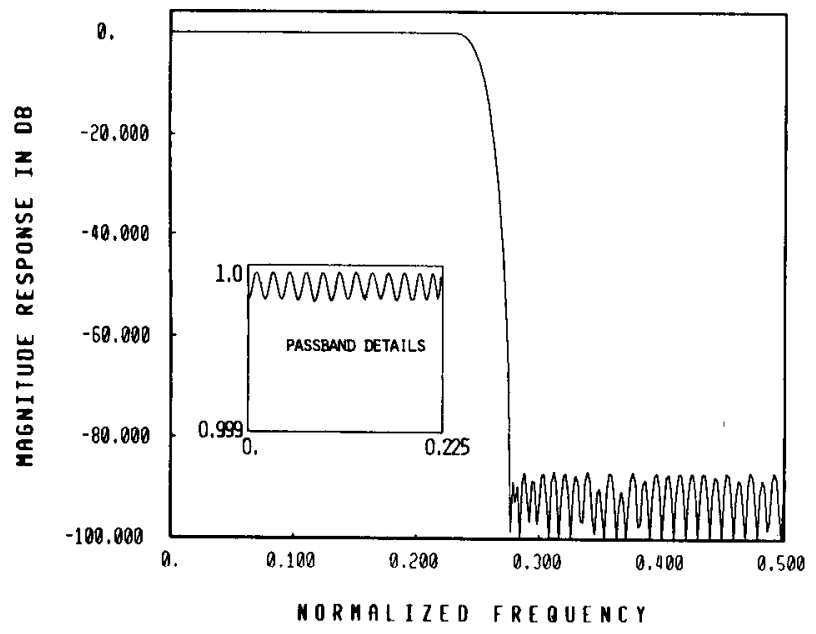

(a)

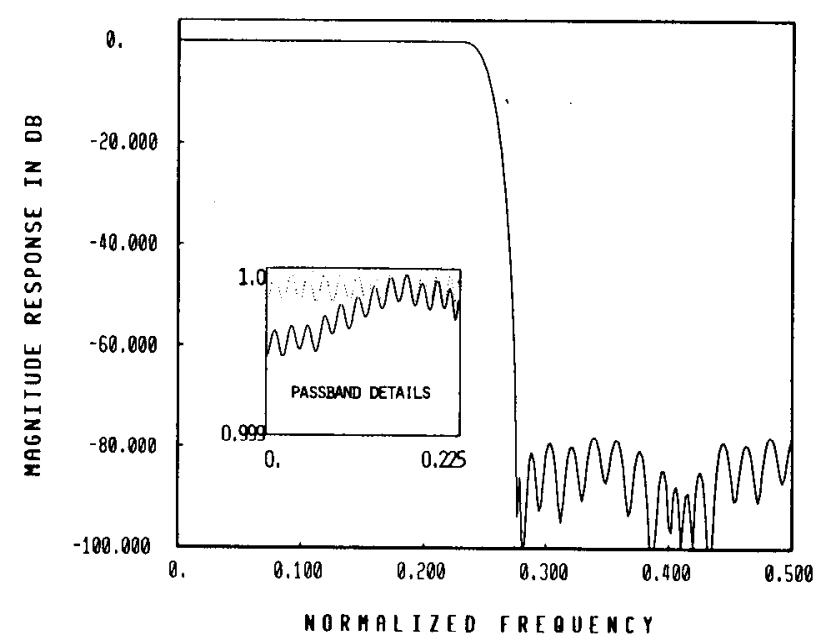

(b)

Fig. 5 (a) Ex. 2: Magnitude response of the half-band filter realized using the lattice structure with 5 bits per multiplier (broken lines denote ideal response). (b) Ex. 2: Magnitude response of the half-band filter realized using the direct form structure with 5 bits per multiplier (broken lines denote ideal response).

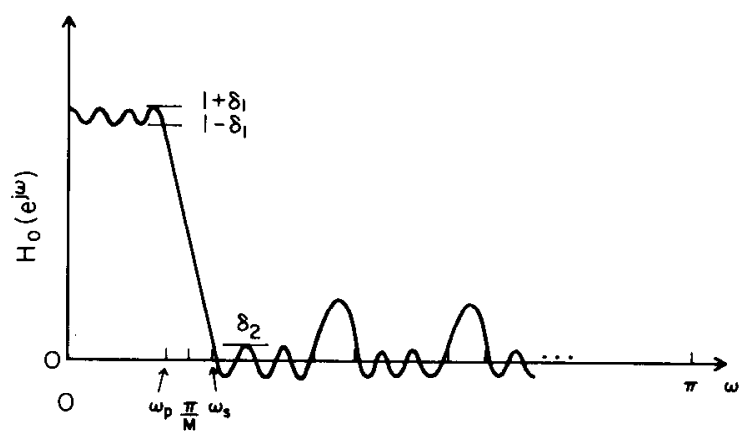

Fig. 6. Typical amplitude response of a $M$ th-band FIR filter.

where $N-1$ is the order. The usual frequency-domain specifications are such that the region $0 \leq \omega \leq \omega_{p}$ represents the passband, and the stopband is interleaved with "don't care" bands. The stopbands typically are defined by

$$
\left[-\omega_{p}+\frac{2 k \pi}{M}, \omega_{p}+\frac{2 k \pi}{M}\right], \quad 1 \leq M-1 .
$$

A direct design of these filters based on judicious use of [5] is outlined in [7]. It is shown there that such a design requires a good guess of the relative values of $\delta_{1}$ and $\delta_{2}$ so that $(6 \mathrm{c})$ is satisfied. Since $(6 c)$ is only approximately satisfied by the resulting design, its coefficients $h(n)$ are readjusted so that $(6 \mathrm{c})$ holds exactly. This results in a loss of equiripple nature. Now, since (6c) prespecifies some of the impulse response coefficients, we can once again, in principle, save design time by eliminating these from the optimization problem. This strategy has the additional advantage that $(6 \mathrm{c})$ is then exactly satisfied.

The natural question that arises here is: "Is there a logical extension of the half-band trick to the $M$ th-band case?" Basically, we wish to first design a related "prototype" function $G(z)$ of lower order (which does not involve the coefficients in $(6 \mathrm{c})$ ) and the construct $H(z)$ from $G(z)$. The first issue is how to formulate such an improved procedure, and secondly, how much, if any, improvements of design time is obtainable? We feel that the answers to these are important to know.

Let us first define a function $V(z)$, related to the $M$ th-band filter $H(z)$ of order $N-1$, as follows:

$$
V(z)=H(z)-\frac{1}{M} z^{-((N-1) / 2)}
$$

Basically, the impulse response $v(n)$ is same as $h(n)$, with the middle term $h((N-1) / 2)$ replaced by zero. Thus

$$
v(n)=0, \quad n-\frac{N-1}{2}=\text { multiple of } M .
$$

Let us represent $V(z)$ in terms of its "polyphase components" [2] as follows:

$$
V(z)=\sum_{k=0}^{M-1} z^{-k} F_{k}\left(z^{M}\right)
$$

so that $v(n)$ is related to the coefficients $f_{k}(n)$ of $F_{k}(z)$ by

$$
f_{k}(n)=v(k+n M), \quad 0 \leq k \leq M-1 .
$$

Because of the constraint $(6 \mathrm{c})$, we conclude that $F_{n_{0}}(z) \equiv 0$, where $n_{0}=N-1 / 2 \bmod M$. We now define the prototype $G(z)$ to be the function, whose impulse response $g(n)$ is constructed from $v(n)$ by eliminating the zero-valued samples in (8). Thus

$$
G(z)=\sum_{k=0}^{n_{0}-1} z^{-k} F_{k}\left(z^{M-1}\right)+\sum_{k=n_{0}}^{M-2} z^{-k} F_{k+1}\left(z^{M-1}\right)
$$

The relation (10) implies

$$
F_{k}(z)=\frac{1}{M} \sum_{l=0}^{M-1}\left(z^{1 / M} W^{-l}\right)^{k} V\left(z^{1 / M} W^{-l}\right)
$$

and therefore

$$
\begin{gathered}
G(z)=\frac{1}{M}\left[\sum_{k=0}^{n_{0}-1} z^{-k} \sum_{l=0}^{M-1}\left(z^{(M-1) / M} W^{-l}\right)^{k} V\left(z^{(M-1) / M} W^{-l}\right)\right. \\
\left.+\sum_{k=n_{0}}^{M-2} z^{-k} \sum_{l=0}^{M-1}\left(z^{(M-1) / M} W^{-l}\right)^{k+1} V\left(z^{(M-1) / M} W^{-l}\right)\right]
\end{gathered}
$$

where $W=e^{j 2 \pi / M}$. The design procedure is now the following: Given the specifications $\omega_{p}$ and $\omega_{S}$ for $H(z)$, find the specifications of $G(z)$ by using the rclation (13). $G(z)$ has a distorted low-pass specification, with cutoff frequencies

$$
\omega_{p}^{\prime}=\omega_{p}\left(\frac{M}{M-1}\right) \quad \omega_{S}^{\prime}=\omega_{S}\left(\frac{M}{M-1}\right)
$$




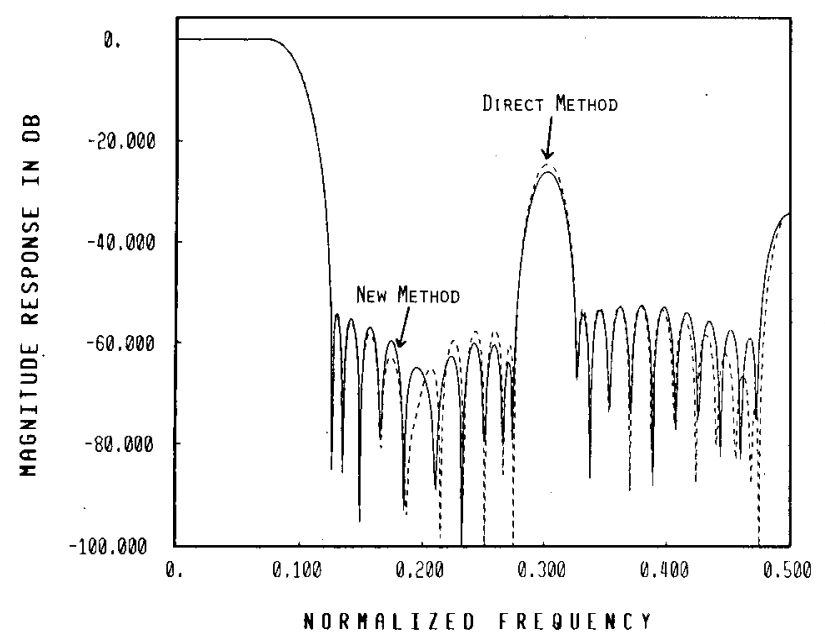

Fig. 7. Ex. 3: Magnitude response of a 5th-band FIR filter designed using the new method and Remez Exchange algorithm.

The attenuation requirement in a given band (for $G(z)$ ) is not a constant any more because of the complicated relationship featured by (13). However, because of the extreme flexibility of the software in [5], $G(z)$ satisfying these specifications can indeed be designed. The impulse response $h(n)$ of the $M$ th-band filter can then be computed from $g(n)$ as

$$
h(n)=\left\{\begin{array}{l}
0, \\
\frac{1}{M}, \\
g\left(n-\left|\frac{n-n_{0}+M}{M}\right|\right),
\end{array}\right.
$$

Example 3: Consider a 5th-band FIR filter of order $N-1=56$ with transition band $\Delta f=0.05$. There are two stopbands (and a "don't care" band) in the region $0 \leq \omega \leq \pi$. We use a relative weighting function $1: 5: 5$ for the designs. Fig. 7 shows the relevant responses for the filter designed using the above method and also by method described in [7].

Table II compares the design time required by the new and conventional methods for $M$ th-band filters with various specifications. Since $G(z)$ has fewer coefficients than $H(z)$, its design is usually faster. However, the complexity involved in mapping the specifications from $H(z)$ to $G(z)$ somctimes reduces the effectiveness of this approach, as seen from the table. Our conclusion is that, for $M=3$, a marginal improvement is obtained by using the above method, whereas for large $M$, the improvement is negligible (and often negative).

\section{CONCLUDING REMARKS}

In this paper, we described an improved method to design equiripple linear-phase FIR half-band and $M$ th-band digital filters. The new design method is considerably faster than the conventional approach for half-band filters. For third-band filters, there is hardly any difference in the design time, as compared to conventional methods. It, however, is slower than the conventional approach for $M>3$ due to additional computations needed in the new method. Since half-band and $M$ th-band FIR filters
TABLE II

Comparison of the New and Conventional Methods in $M$ Th Band Filter Designs

\begin{tabular}{|c|c|c|c|c|c|c|c|c|}
\hline \multirow[b]{2}{*}{$N-1$} & \multirow[b]{2}{*}{$M$} & \multirow[b]{2}{*}{$\Delta f$} & \multicolumn{3}{|c|}{ NEW METHOD } & \multicolumn{3}{|c|}{ DIRECT METHOD } \\
\hline & & & $\delta_{1}$ & $\delta_{2}$ & $\underset{\text { (eec.) }}{\text { CPU Time }}$ & $\delta_{1}$ & $\delta_{2}$ & $\underset{\text { (esc.) }}{\text { CPU Time }}$ \\
\hline 88 & 3 & .025 & 0.00593 & 0.00683 & 13.7 & 0.00810 & 0.00816 & 14.8 \\
\hline 100 & 3 & .025 & 0.00365 & 0.00401 & 16.4 & 0.00490 & 0.00478 & 21.6 \\
\hline 124 & 3 & .025 & 0.00130 & 0.00143 & 24.6 & 0.00173 & 0.00158 & 26.4 \\
\hline go & 4 & .025 & 0.00625 & 0.00735 & 22.3 & 0.00460 & 0.00784 & 11.6 \\
\hline 126 & 4 & .025 & 0.00118 & 0.00120 & 33.6 & 0.00126 & 0.00126 & 28.8 \\
\hline 78 & 5 & .025 & 0.00950 & 0.01070 & 25.4 & 0.01085 & 0.01120 & 13.5 \\
\hline 126 & 6 & .025 & 0.00112 & 0.00140 & 52.8 & 0.00111 & 0.00157 & 20.1 \\
\hline
\end{tabular}

are widely used in signal decimation, interpolation, filtering, and also in the minimization of intersymbol interference [11], the improved designs presented above are expected to be of wide interest.

\section{ACKNOWLEDGMENT}

The authors wish to thank $\mathrm{Z}$. Dog̃anata for generating the example on low-sensitivity half-band filter design.

$$
\begin{aligned}
& n-\frac{N-1}{2}=\text { nonzero multiple of } M \\
& n=\frac{N-1}{2} \\
& \text { otherwise. }
\end{aligned}
$$

\section{REFERENCES}

[1] M. G. Bellanger, J. L. Daguet, and G. P. Lepagnol, "Interpolation, extrapolation and reduction of computation speed in digital filters," IEEE Trans. Acoust., Speech, Signal Process., vol. ASSP-22, pp. 231-235, Aug. 1974.

[2] R. E. Crochiere and L. R. Rabiner, Multirate Digital Signal Processing. Englewood Cliffs, NJ: Prentice Hall, 1983.

[3] M. J. T. Smith and T. P. Barnwell III, "A procedure for designing exact reconstruction filter banks for tree structured subband coders," in Proc. IEEE Int. Conf. Acoust., Speech, Signal Process., (San Diego, CA), Mar. 1984, pp. 27.1.1-27.1.4

[4] F. Mintzer, "Filters for distortion-free two-band multirate filter banks," IEEE Trans. Acoust., Speech, Signal Process., vol. ASSP-33, pp. 626-630, June 1985 .

[5] J. H. McClellan, T. W. Parks, and L. R. Rabiner, "A computer program for designing optimum FIR linear phase digital filters," IEEE Trans. A udio Electroacoust., vol. AU-21, pp. 506-526, Dec. 1973.

[6] O. Herrmann and W. Scheussler, "On the accuracy problem in the design of nonrecursive digital filters," Arch. Elek. Übertragung., vol. 24, pp. 525-526, 1970.

[7] F. Mintzer, "On half-band, third-band and $N$ th band FIR filters and their design," IEEE Trans. Acoust, Speech, Signal Process, vol. ASSP30, pp. 734-738, Oct. 1982.

[8] P. P. Vaidyanathan and S. K. Mitra, "Low passband sensitivity digital filters; A generalized viewpoint and synthesis procedures," Proc. IEEE, vol. 72, pp. 404-423, Apr. 1984.

[9] D. S. K. Chan and L. R. Rabiner, "Analysis of quantization errors in the direct form for finite impulse response digital filters," IEEE Trans. Audio Electroacoust., vol. AU-21, pp. 354-366, Aug. 1973.

$[10\rceil$ P. P. Vaidyanathan, "New cascaded lattice structure for FIR filters having extremely low coefficient sensitivity," in Proc. IEEE. Int. Conf. A coust., Speech, Signal Process. (Tokyo, Japan), Apr. 1986, pp. 497-450.

[11] K. Nakayama and T. Mizukami, "A new IIR Nyquist filter with zero intersymbol interference and its frequency response approximation," IEEE Trans. Circuits Syst., vol. CAS-29, pp. 23-34, Jan. 1982.

[12] L. R. Rabiner and B. Gold, Theory and Application of Digital Signal Processing. Englewood Cliff, NJ: Prentice Hall, 1975. 